\title{
Influenza Vaccination in Pregnancy: Current Practices in a Suburban Community
}

\author{
David H. Wallis, MD, Jennifer L. Chin, MD, and Denise K. C. Sur, MD
}

Purpose: The Centers for Disease Control (CDC) identify women in their second and third trimesters as a high-risk population warranting influenza vaccination. This study attempted to characterize understanding of these guidelines and obstacles to their implementation in a suburban community.

Methods: Family physicians and obstetricians with admitting privileges to a community-based hospital were surveyed regarding estimated vaccine availability and administration in their practices and regarding knowledge of indications and contraindications to influenza vaccination in pregnancy.

Results: Of the 20 obstetricians and 66 family physicians completing the survey, $68.4 \%$ of obstetricians and $90.5 \%$ of family physicians carried the vaccine in their offices $(P=.027)$. Both obstetricians and family physicians incorrectly perceived multiple factors as contraindications to influenza vaccination in pregnancy. Obstetricians and family physicians reported similar proportions of their pregnant patients received the vaccine ( 35 versus $40 \%$ ).

Conclusions: In this study, more family physicians had the influenza vaccine available than obstetricians, but there was no difference in estimated rates of vaccination during pregnancy or in the understanding of its indications and contraindications. Finally, no physicians in our community reported providing influenza vaccination in pregnancy at recommended frequencies. Further research is needed to clarify methods of improving vaccination rates in both family practice and obstetric settings. ( $\mathrm{J}$ Am Board Fam Pract 2004;17:287-91.)

Every year, the influenza virus affects $10 \%$ to $20 \%$ of the US population, with hundreds of thousands of patients hospitalized because of influenza infection and complications. ${ }^{1}$ Each epidemic results in more than 100,000 hospitalizations and more than 20,000 deaths. ${ }^{2,3}$ The influenza virus constantly undergoes genetic recombination of the coding for surface antigens. Point mutations result in minor structural changes (antigenic drift) of these proteins, whereas genetic recombination resulting in more major alterations in surface antigens (antigenic shift) clinically accounts for major periodic epidemics of disease. Both frustrate the development of immunity on an individual or global scale.

Aerosolized respiratory secretions transmit the influenza virus. These secretions attach to respira-

Submitted, revised, 26 January 2004.

From the Santa Monica-UCLA Family Practice Residency Program, David Geffen School of Medicine at University of California, Los Angeles. Address correspondence to David H. Wallis, MD, Beach Cities Medicine, 703 Pier Ave, Suite B708, Hermosa Beach, CA 90254 (E-mail: drwallis@beachcitiesmedicine.com).

This work was supported financially by a research stimulation grant from the Joint American Academy of Family Physicians/F-AAFP Grant Awards Council. Manuscript content was presented in poster format at the AAFP Scientific Assembly; 2002 Oct; San Diego, California. tory epithelium and are shed for 5 to 10 days after an incubation period of 1 to 5 days. Confounding efforts to contain the disease, the period of maximal communicability may start 1 to 2 days before symptom onset. Classic symptoms of fever, myalgia, cough, and headache are seen in only about $50 \%$ of infections, depending on prior immunologic exposure to antigenically similar viruses and other aspects of the host's general condition. Although the duration of illness generally does not exceed 3 to 5 days, some may experience prolonged symptoms of cough, malaise, and fatigue for weeks. ${ }^{4}$

In addition to being a frequent cause of morbidity, influenza infection often contributes to mortality from associated complications. The elderly and those with pre-existing pulmonary disease are particularly susceptible to secondary bacterial pneumonia. Influenza infection also often exacerbates long-term health problems, carrying significant morbidity and mortality implications as well. Although less common, primary influenza pneumonia carries a high case-fatality rate. Other conditions, such as Reye syndrome and myocarditis, are much more rare.

Because numerous problems surround efforts to stop the spread of the virus through isolation or 
treatment of infected persons, most prevention efforts focus on immunization of at-risk populations. Because of antigenic drift of the influenza virus, the vaccine is reformulated annually to contain inactivated antigens of the strains deemed most likely to cause infection in the coming year. Although efficacy of the vaccine varies widely with host and environmental factors, it is up to $90 \%$ effective in preventing disease among healthy, immunocompetent adults. ${ }^{5}$ Even among the elderly, it is approximately $60 \%$ effective in preventing hospitalizations. ${ }^{6}$ Adverse reactions are mild, with $10 \%$ to $64 \%$ experiencing limited local reactions (soreness or redness) but other symptoms (including fever, myalgia, headache, or fatigue) occurring no more often than with placebo. ${ }^{4,7}$

Whereas $40 \%$ to $50 \%$ of hospitalizations and $90 \%$ of deaths occur in patients over 65 years old, studies have also demonstrated increased risk of complications in younger patients residing in longterm care facilities; those with chronic diseases, such as asthma; and pregnant women. ${ }^{4}$ Efficacy is maximized if the vaccine is administered 2 weeks before exposure to the virus. ${ }^{8}$ The Centers for Disease Control (CDC) identified at-risk populations for the 2003 to 2004 influenza season (Table 1), eliminating the previously accepted tiered fashion of vaccination prioritization.

Women in their second and third trimesters of pregnancy have an increased risk of influenzarelated complications including pneumonia and a 4-fold risk of hospitalization. ${ }^{9}$ Because these hospitalization rates are comparable with those of nonpregnant women with high-risk medical conditions, the CDC has adopted similar guidelines regarding vaccination for both groups. Women who will be in the second or third trimester during influenza season and all pregnant women with additional high-risk medical conditions should receive vaccination in the fall. Some advocate avoidance of vaccination during the first trimester and instead vaccinate during preconception planning or after 14 weeks' gestation. ${ }^{4}$ The first trimester poses no significant increase from baseline in the risk of influenza-related complications, and routine administration of any vaccine during this time may prompt anecdotal association with naturally occurring spontaneous miscarriage. Adverse effects from influenza vaccination are uncommon. Localized soreness is frequently noted; infrequent mild symptoms, such as fever, malaise, and myalgias, are re-
Table 1. Candidates for Influenza Vaccination

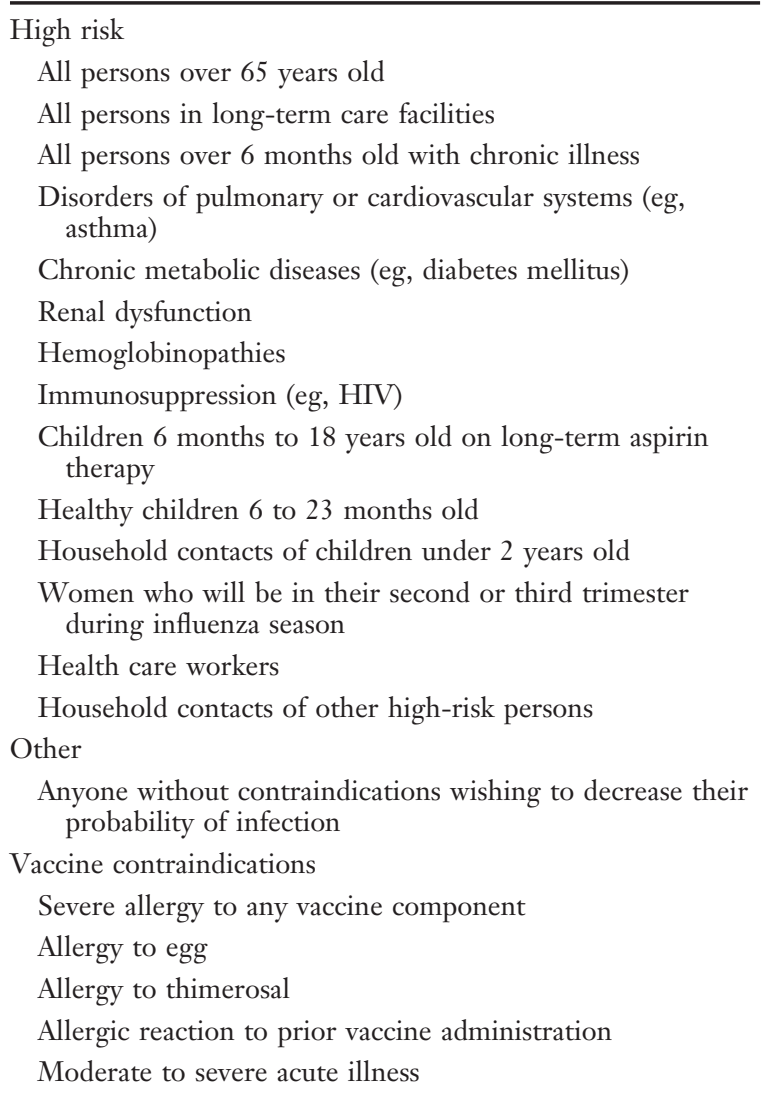

ported with frequency similar to that of placebo injection. These symptoms usually occur after the initial vaccination and last only 1 to 2 days. Breastfeeding is not a contraindication to vaccination.

Despite publication of these guidelines, rates of vaccination among high-risk patients remain low. ${ }^{10-12}$ Many possible explanations exist for this discrepancy, including vaccine unavailability, logistic concerns, poor reimbursement, fear of side effects, and lack of adequate patient or physician education. This survey tool was designed to assess physicians' individual practice styles and awareness of CDC recommendations for influenza vaccination during pregnancy. It is our hope that by characterizing the understanding and application of current knowledge to patient care, obstacles can be identified, and more effective interventions can be designed to increase rates of vaccination among pregnant women in the community.

\section{Methods}

The study population comprised all family physicians and obstetricians with admitting privileges at 
Santa Monica-UCLA Medical Center in Santa Monica, California. A survey was designed to ascertain the general knowledge of the indications and contraindications to influenza vaccination in pregnancy, as well as the estimated availability and frequency of administration of the vaccine in the participating physicians' offices (survey available on request). This anonymous survey was mailed to each physician in December 2001, with a second "reminder" mailing sent approximately 1 month later.

The survey inquired about the availability of the influenza vaccine in the physicians' offices during the influenza season and whether the physicians administer the vaccine during pregnancy. The survey also assessed whether various maternal conditions were deemed relative indications or contraindications to influenza vaccination during pregnancy. Last, physicians were asked to estimate the frequencies with which the influenza vaccine is requested and received by pregnant patients in their practices.

Responses were tallied by a blinded third party. Statistical analysis was performed; $p$ values were calculated by Fisher's exact or $t$ test as appropriate and equal variance assumed within practices for continuous variables.

\section{Results}

A total of 184 physicians met entry criteria: 67 obstetricians (36.4\%) and 117 family physicians (63.6\%). After the initial and reminder mailings, 99 responses $(53.8 \%)$ were received. Of these, 20 were obstetricians (20.2\%) and 66 were family physicians (66.7\%). The remaining 13 responses were gynecological subspecialists or did not specify their practice type and were excluded. Not every physician answered all questions polled on our survey, which was taken into account during statistical analysis.

\section{Availability and Administration of the Influenza Vaccine}

Significantly fewer obstetricians reported influenza vaccine availability in their office than family physicians $(68.4 \%$ versus $90.5 \% ; P=.027)$. There was no significant difference between obstetricians and family physicians with regard to whether they administer the influenza vaccine during pregnancy (79\% versus $72 \% ; P=.406$ ), as shown in Figure 1 .

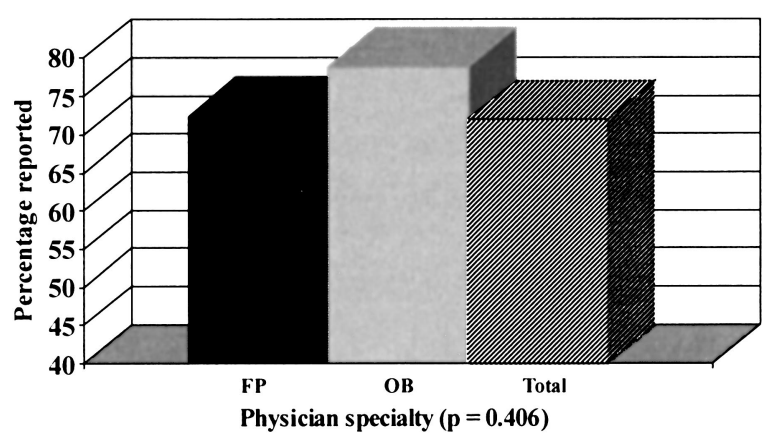

Figure 1. Administration of influenza vaccine during pregnancy as reported by practice type. FP: family physician; OB: obstetrician.

\section{Perceived Contraindications}

The survey was designed to ascertain physicians' views on suggested relative contraindications to administering the influenza vaccine during pregnancy. The maternal conditions surveyed were as follows: first trimester, second trimester, third trimester, gestational diabetes, pregnancy-induced hypertension, advanced maternal age, maternal heart disease, maternal lung disease, history of preterm labor, and history of intrauterine fetal demise. No significant difference was found between the responses of the two comparison groups, with $p$ values for each comparison $>0.05$ (Table 2).

\section{Request for and Receipt of Influenza Vaccine in Pregnancy}

The estimated frequency of request or receipt of the influenza vaccine during pregnancy was reported by physicians as a continuous variable. As shown in Figure 2, 26.9\% of pregnant women requested an influenza vaccine from their obstetrician compared with $17.1 \%$ who requested vaccination from their family physician $(P=.051$, by $t$ test for equality of means). Whether administered by their family physician, obstetrician, or other health care provider, obstetricians reported that $34.5 \%$ of their pregnant patients received the influenza vaccine, whereas family physicians reported $40.1 \%$ ( $P=$ $.453)$.

\section{Discussion}

This study demonstrates that although family physicians in our community are more likely than obstetricians to carry the influenza vaccine in their offices, there is no statistically significant difference in the frequency of vaccine administration during 
Table 2. Perceived Relative Contraindications to the Administration of Influenza Vaccine during Pregnancy

\begin{tabular}{|c|c|c|c|c|}
\hline \multirow[b]{2}{*}{ Condition } & \multicolumn{2}{|c|}{ 'Relative Contraindication' Response } & \multirow[b]{2}{*}{ Overall } & \multirow[b]{2}{*}{$P$ value } \\
\hline & Family Physicians & Obstetricians & & \\
\hline First trimester & $63.2 \%(36 / 57)$ & $64.7 \%(11 / 17)$ & $63.5 \%$ & .573 \\
\hline Second trimester & $5.4 \%(3 / 56)$ & $0 \%(0 / 17)$ & $4.1 \%$ & .446 \\
\hline Third trimester & $8.8 \%(5 / 57)$ & $0 \%(0 / 18)$ & $6.7 \%$ & .243 \\
\hline Gestational diabetes & $3.6 \%(2 / 56)$ & $0 \%(0 / 17)$ & $2.7 \%$ & .586 \\
\hline Pregnancy-induced hypertension & $11.1 \%(6 / 54)$ & $6.7 \%(1 / 15)$ & $10.1 \%$ & .523 \\
\hline Advanced maternal age & $5.8 \%(3 / 52)$ & $0 \%(0 / 17)$ & $4.3 \%$ & .422 \\
\hline Maternal heart disease & $1.7 \%(1 / 58)$ & $0 \%(0 / 19)$ & $1.3 \%$ & .753 \\
\hline Maternal lung disease & $1.7 \%(1 / 58)$ & $0 \%(0 / 19)$ & $1.3 \%$ & .753 \\
\hline History of preterm labor & $15.7 \%(8 / 51)$ & $5.9 \%(1 / 17)$ & $13.2 \%$ & .281 \\
\hline History of intrauterine fetal demise & $14.6 \%(7 / 48)$ & $0 \%(0 / 15)$ & $11.1 \%$ & .133 \\
\hline
\end{tabular}

pregnancy. Although our data suggest that obstetricians may field more requests for the influenza vaccine from their pregnant patients, there was no statistically significant difference between the two practice types in the reported receipt of the vaccine. One possible explanation for these results is that family physicians, who are generally well-versed in preventive services such as immunizations, may be more likely to offer vaccines in their offices but also may become overly wary of administering vaccines during pregnancy. Conversely, obstetricians may not carry the vaccine as frequently because of logistic or other concerns.

Epidemiologic data demonstrate the peak of influenza season occurring in the 4-month period between December and March over $90 \%$ of the last 25 years. ${ }^{4}$ Because the second and third trimesters encompass 26 weeks' time, only $17 \%$ of pregnant women would not be expected to have an increased risk during the peak season, thus not warranting immunization. This model does not take into ac-

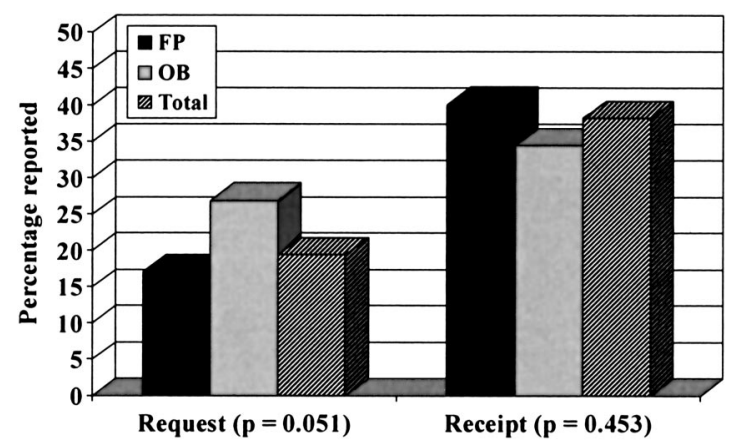

Figure 2. Frequencies of patient request and receipt of influence vaccination during pregnancy as reported by practice type. FP: family physician; OB: obstetrician. count seasonal variations in birth rates or exclusion because of history of allergy to a vaccine component. Nonetheless, based on these estimates and CDC recommendations, one might anticipate a goal immunization rate of greater than $70 \%$ of this high-risk population. In contrast, both family physicians and obstetricians reported administering the influenza vaccine to less than $40 \%$ of their pregnant patients, demonstrating the need for appropriate interventions to increase influenza vaccination rates in pregnancy.

A number of maternal conditions were perceived as potential contraindications to influenza vaccination during pregnancy. The most common of these were the first trimester, history of preterm labor, history of intrauterine fetal demise, and pregnancyinduced hypertension; none of these are listed by the CDC as contraindications. According to CDC guidelines, the only absolute contraindications to the influenza vaccine during pregnancy are a history of allergy to its components and a moderate to severe febrile illness. Furthermore, although some may consider the first trimester a period of relative contraindication, even this administration has shown no adverse maternal or fetal effects. ${ }^{4}$

Another potentially significant obstacle to influenza vaccination during pregnancy was physician reimbursement. Several responders remarked that reimbursement from insurance companies played a part in whether they stocked the vaccine in their offices and whether it was administered to pregnant patients. Although they acknowledged the indications for the vaccine, some obstetricians stated that insurance plans have refused reimbursement for vaccination because they were not the patient's 
primary care provider for this "preventive" service. Although patients may still be instructed to obtain vaccination elsewhere, this additional obstacle to recommended obstetrical care may result in lower immunization rates.

One limitation of this study is that it surveyed only physicians with admitting privileges to one community hospital, with limited numbers and unknown applicability to other practice environments. Some physicians with privileges may not be actively practicing obstetrics, so the survey responses may not precisely reflect current practice in the area. Local variations may also limit generalizability. The possibility of a response bias also cannot be excluded. Finally, the survey involved selfreporting of vaccination rates rather than directed chart reviews, introducing a possible source of inaccuracy.

In conclusion, this study found no difference between family physicians and obstetricians with regard to their understanding of the indications and contraindications to influenza vaccination during pregnancy but did demonstrate a significant difference between them in the availability of the vaccine in their offices. It also raises the possibility of reimbursement restrictions as a significant obstacle to recommended vaccination among pregnant women, suggesting an area for policy improvements in health care. In addition, this study demonstrates discrepancies, similar to those reported in other studies, ${ }^{10,11}$ between CDC recommendations for influenza vaccination during pregnancy and the reported frequency of administration. Further research is required to determine effective methods of increasing vaccination rates in this high-risk population, which may include educational seminars, chart reminders, or other interventions.

\section{References}

1. Dolin R. Infectious disease. In: Braunwald E, et al, editors. Harrison's principles of internal medicine. 15th ed. New York: McGraw-Hill; 2001. p. 112530.

2. Simonsen L, Fukuda K, Schonberger LB, Cox NJ. Impact of influenza epidemics on hospitalizations. J Infect Dis 2000;181:831-7.

3. Simonsen L, Clarke MJ, Schonberger LB, Arden NH, Cox NJ, Fukuda K. Pandemic versus epidemic influenza mortality: a pattern of changing age distribution. J Infect Dis 1998;178:53-60.

4. Bridges CB, Harper SA, Fukuda K, Uyeki TM, Cox NJ, Singleton JA; Advisory Committee on Immunization Practices. Prevention and control of influenza. Recommendations of the Advisory Committee on Immunization Practices (ACIP) [published erratum appears in MMWR Morb Mortal Wkly Rep 2003; 52:526]. MMWR Recomm Rep 2003;52(RR-8):134; quiz CE1-4.

5. Zimmerman RK, Middleton DB, Burns IT, Clover RD. Routine vaccines across the life span, 2003. J Fam Pract 2003 (1 Suppl);52:S1-21.

6. Patriarca PA, Weber JA, Parker RA, et al. Efficacy of influenza vaccine in nursing homes. Reduction in illness and complications during an influenza A (H3N2) epidemic. JAMA 1985;253:1136-9.

7. Bridges CB, Thompson WW, Meltzer MI, et al. Effectiveness and cost-benefit of influenza vaccination of healthy working adults. A randomized, controlled trial. JAMA 2000;284:1655-63.

8. Bridges CB, Fukuda K, Cox NJ, Singleton JA; Advisory Committee on Immunization Practices. Prevention and control of influenza. Recommendations of the Advisory Committee on Immunization Practices (ACIP). MMWR Recomm Rep 2001;50(RR-4): $1-44$.

9. Neuzil KM, Reed GW, Mitchel EF, Simonsen L, Griffin MR. Impact of influenza on acute cardiopulmonary hospitalizations in pregnant women. Am J Epidemiol 1998 Dec 1;148:1094-102.

10. Silverman NS, Greif A. Influenza vaccination during pregnancy. Patients' and physicians' attitudes. J Reprod Med 2001;46:989-94.

11. Schrag SJ, Fiore AE, Gonik B, et al. Vaccination and perinatal infection prevention practices among obstetrician-gynecologists. Obstet Gynecol 2003;101: 704-10.

12. Szilagyi P, Vann J, Bordley C, et al. Interventions aimed at improving immunization rates. Cochrane Database Syst Rev 2002;(4):CD003941. 